\title{
Continuité des flux hydriques dans le système sol-racines-tronc. Cas des arbres fruitiers
}

\author{
B Cabibel \\ avec la participation technique de $\mathrm{J}$ Horoyan \\ INRA, unité de science du sol, domaine Saint-Paul, BP 91, F84143 Montfavet cedex, France
}

(Reçu le 15 septembre 1994 ; accepté le 2 novembre 1994)

\begin{abstract}
Résumé - L'analyse des flux de sève, mesurés par voie thermique, dans les racines et le tronc d'un pommier, couplée à l'étude des transferts de l'eau dans le sol, est utilisée pour étudier le transfert de celle-ci dans le système sol-plante. On montre qu'il existe une hétérogénéité des flux dans le tronc et entre les différents faisceaux racinaires de l'arbre, ces hétérogénéités étant bien corrélées. Leurs amplitudes dépendraient de l'hétérogénéité de densité racinaire en sol hydriquement homogène et en sol hydriquement hétérogène de l'hétérogénéité d'humectation locale des différents horizons du sol. La répartition spatiale des pertes en eau journalières mesurées dans le sol étant de plus bien corrélée à la structure spatiale des extractions d'eau par les racines primaires de l'arbre, on en conclut qu'il existe une continuité des flux entre un secteur de sol donné, le faisceau racinaire qu'il contient et le secteur du tronc correspondant au faisceau racinaire.
\end{abstract}

Malus = pommier $/$ flux de sève $/$ mesure thermique $/$ continuité des transferts hydriques $/$ sol $/$ racine $/$ tronc

Summary - Continuity of water transfer in soil-plant system: the case of fruit trees. A thermal method for measuring sap flow in apple-tree trunks and roots, combined with a water-soil transfer study has been utilized to investigate water transfer in a soil-plant system. It has been shown that: i) heterogeneities of sap flux are present in apple-tree trunk and root systems; ii) these heterogeneities are closely correlated and induced either by the heterogeneity of root density in moistened homogeneous soil or by the heterogeneity of different soil horizon wetting in moistened heterogeneous soil; and iii) the spatial distribution of daily water-soil loss is in agreement with the spatial distribution of water-soil extraction by the different root bundles. It has been concluded that a continuous flow exists between one soil sector, the root bundle present in that soil sector, and the trunk sector corresponding to the root bundle.

Malus = apple tree / sap flow / thermal measurement / water transfer continuity / soil / root / trunk

\section{INTRODUCTION}

On admet généralement que la croissance, le développement et le fonctionnement des systèmes racinaires des plantes annuel.les sont étroitement liés au mode d'irrigation èt que leur consommation en eau est fortement dépendante de la densité moyenne et de l'homogénéité de distribution de leurs racines dans le profil de sol (Lo Cascio, 1980 ; Ruggiero, 1980 ; Sanders et al, 1989). Ces 2 paramètres, qui règlent le trajet moyen pour l'eau jusqu'aux sites d'extraction et le niveau d'exploitation du volume global de l'eau disponible, déterminent en effet le degré de satisfaction de la demande climatique s'exerçant sur la culture. 
Inversement, pour les plantes pérennes, et notamment pour les arbres fruitiers à pépins (pommiers), on a montré (Cabibel, 1978 ; Cabibel, 1991 ; Habib, 1978) qu'il n'existe, sous irrigation localisée de complément, aucune relation stricte entre l'irrigation et le développement des systèmes racinaires, alors même que leur fonctionnement est totalement régi par l'hétérogénéité de répartition de l'eau apportée dans la zone de sol humectée.

L'extraction de l'eau par le système racinaire des arbres ne peut donc, sous irrigation localisée, être quantifiée à l'aide des normes classiques de caractérisation que sont la biomasse et la densité moyenne de répartition des racines dans le profil de sol. Pour un système sol-plante-climat donné, les potentialités d'extraction racinaire pour l'eau devront être alors explicitées à la fois en termes de quantité par évaluation du flux global traversant l'arbre et en termes de localisation dans le sol, par établissement de la fonction de distribution de l'intensité de l'absorption racinaire par rapport au point d'apport de l'eau.

Dans ce qui suit nous présentons des résultats qui caractérisent, avant irrigation, le fonctionnement du système racinaire des arbres, les transferts de sève brute dans le tronc et les relations existant entre les différents flux hydriques élémentaires dans le sol, le système racinaire et le tronc de l'arbre.

\section{MATÉRIEL ET MÉTHODES}

\section{Le verger expérimental}

II est constitué de pommiers Golden Delicious greffés sur MM106. Plantés dans un sol d'alluvions récentes argilo-calcaires de la Durance, les arbres sont disposés en quinconce selon une maille de $8 \times 5 \mathrm{~m}$. Ils sont irrigués depuis leur plantation en 1979, d'abord par aspersion, puis ponctuellement par ajutage Bas-Rhone depuis 4 ans. L'étude est conduite sur un arbre dont les caractéristiques morphologiques et végétatives (circonférence du tronc, développement végétatif, allongement des rameaux, récoltes antérieures) ont pour valeurs celles trouvées statistiquement moyennes pour l'ensemble des arbres du verger (Cabibel et Do, 1991a). Le volume total de sol attribué à l'arbre, dont la trace en surface est un hexagone de $30 \mathrm{~m} 2$ d'aire, est isolé latéralement du reste du sol du verger par une barrière imperméable, un film plastique très épais ayant été descendu dans une tranchée sur $2 \mathrm{~m}$ de profondeur (fig 1). En conséquence ce volume de sol n'est accessible qu'au système racinaire de l'arbre isolé et ses variations de teneur en eau ne peuvent être le fait que de l'extraction racinaire de l'arbre test et de l'évaporation directe du sol.

\section{Méthodes de mesures}

La quantification des transferts liquides dans le système sol-plante est accessible par analyse simultanée

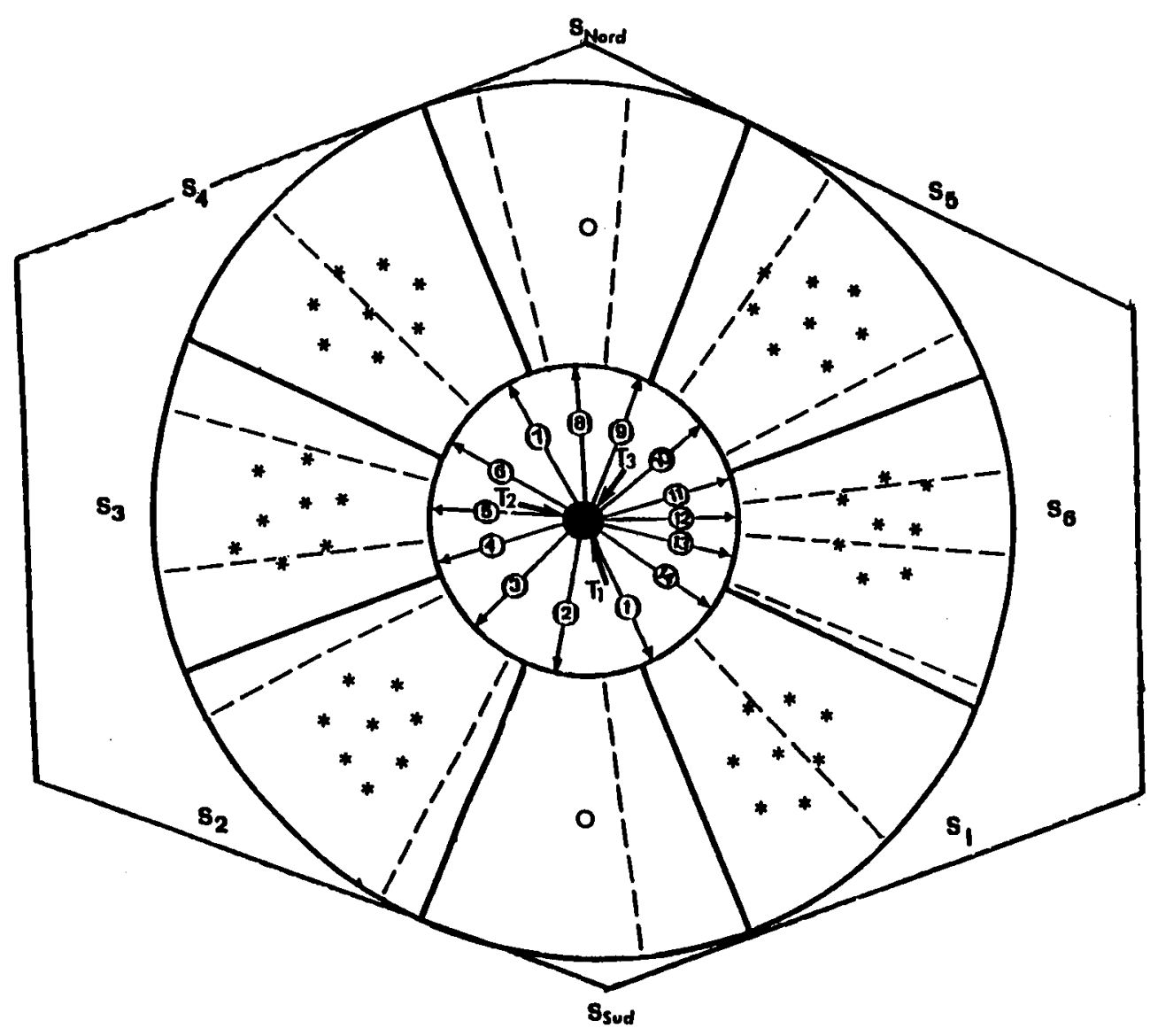

Fig 1. Dispositif expérimental. - - Limite secteur d'exploration racinaire ; - Limite secteur sol $45^{\circ} ; \leftarrow 0-$ Direction des racines; o tube d'accès pour sonde à neutron ; * tensiomètre ; $\leftarrow$ T Fluxmètre dans le tronc ( $T 1$, T2, T3). 
du bilan de l'eau dans le sol et des transferts de sève brute dans les différents organes de l'arbre. L'estimation de la fonction puits racinaire totale sera obtenue par mesure du flux global traversant le tronc et sa fonction de répartition dans le sol par mesure des flux élémentaires dans chacune des racines principales.

\section{Le climat}

Les paramètres climatiques (humidité relative de l'air, rayonnements net et global, vitesse du vent) nécessaires au calcul de l'ETP Penman sont mesurés par l'unité de bioclimatologie de I'INRA-Avignon sur une parcelle contiguë au verger expérimental. L'ETP horaire est obtenue à l'aide d'un logiciel élaboré par le service technique d'études des facteurs climatiques de l'environnement de l'INRA-Avignon.

\section{Quantification des transferts d'eau dans le sol}

Le suivi des teneurs en eau, $\theta\left(z_{n}, t\right)$, est assuré par humidimétrie neutronique. On dispose de 2 tubes d'accès pour une sonde humidimètre-densimètre CPN. L'étalonnage neutronique, de type gravimétrique, a porté sur les couches de sol $0-15 \mathrm{~cm}$ et $25-165 \mathrm{~cm}$. Le pas vertical de mesure est de $10 \mathrm{~cm}$. La précision sur la mesure de $\theta$ est estimée à $\pm 0,015 \mathrm{~m}^{3}$ d'eau $/ \mathrm{m}^{3}$ de sol. Le suivi des potentiels de l'eau du sol, $h\left(z_{n}, t\right)$, est effectué à l'aide de 48 tensiomètres répartis en 6 sites de mesures. Les potentiels sont mesurés, dans chaque site, à $20,35,50,65,80,100,150$ et $200 \mathrm{~cm}$ de profondeur. Une mesure journalière est effectuée à $8 \mathrm{~h}$ GMT à l'aide d'une sonde DTE 1000. La précision des mesures sur le potentiel est estimée à $\pm 5 \mathrm{hPa}$. Les dispositifs de mesure sont implantés sur une circonférence de $150 \mathrm{~cm}$ de rayon centrée sur l'arbre (fig 1). Les relations $h(\theta)$, en humectation et en dessiccation, ont été déterminées in situ (Bon, 1986).

Les variations de stock hydrique sont calculées, entre 20 et $200 \mathrm{~cm}$ de profondeur, dans les 8 secteurs du sol de $45^{\circ}$, chaque secteur étant centré sur un dispositif de mesures tensiométrique ou neutronique (fig 1). La limite inférieure du profil de sol étudié est imposée par la profondeur du système de mesure. La limite supérieure a été fixée à la suite d'une étude préalable in situ du système racinaire de plusieurs arbres du verger, qui a mis en évidence l'absence totale de racines dans l'horizon $0-25 \mathrm{~cm}$. Toutes variations de stock d'eau pouvant être trouvées dans l'horizon 0-20 $\mathrm{cm}$, lors de la période d'établissement des bilans, ne pouvaient dès lors être attribuées à une extraction racinaire, et ce d'autant qu'aux teneurs en eau initiales mesurées dans cet horizon, toujours inférieures à $0,21 \mathrm{~m}^{3}$ d'eau $/ \mathrm{m}^{3}$ de sol, correspondaient des potentiels supérieurs à ceux au point de flétrissement permanent. Toute perte en eau dans cet horizon de sol n'étant dès lors attribuable qu'à l'évaporation du sol nu, elle ne pouvait être prise en compte dans l'estimation de la fonction puits racinaire de l'arbre. On a négligé les remontées capillaires vers la couche $200 \mathrm{~cm}$ et les transferts diffusifs verticaux vers l'hori- zon de surface du fait de la très faible variation des teneurs en eau dans les horizons immédiatement sous jacents et de la très faible conductivité hydraulique de ce sol dans la gamme des teneurs en eau présentes (Bon, 1986).

Dans les 2 secteurs nord et sud, les bilans sont établis à partir des mesures neutroniques. Dans les 6 autres secteurs, ils le sont à partir des teneurs en eau volumiques déduites de la relation $h(w)$. L'utilisation de cette relation suppose vérifiée l'hypothèse de son homogénéité spatiale sur le site expérimental. Le calcul de $\theta$ ne repose alors que sur un changement de variable unique, la densité apparente des différents horizons du sol. Les potentiels mesurés dans la couche $20-50 \mathrm{~cm}$ étant en valeur absolue au-delà de la gamme de mesure possible, on a affecté à cette couche de sol dans les 6 bilans tensiométriques, la variation de stock moyenne trouvée par mesure neutronique sur les 2 sites Nord et Sud. Pour la séquence de temps étudiée (2-5 juillet), cette variation de stock moyenne est trouvée égale à $0,31 \mathrm{~mm}$ par jour, les valeurs nord et sud étant respectivement de 0,28 et $0,35 \mathrm{~mm}$ par jour.

\section{Quantification des transferts de sève brute dans l'arbre}

\section{Principe de la mesure}

La mesure des flux de sève brute est réalisée par voie thermique selon la méthode du flux radial (Granier, 1985). Le flux de sève total $U$ est égal au produit de la densité du flux Ju par la surface conductrice du bois $\mathrm{S}$ estimée dans le plan de la mesure.

$$
\mathrm{U}=\mathrm{Ju} . \mathrm{S}
$$

Ju étant exprimée en $\mathrm{m}_{\mathrm{s}} \mathrm{s}^{-1}$ et $\mathrm{S}$ en $\mathrm{m}^{2}$, $\mathrm{U}$ s'exprime en $\mathrm{m}^{3} \cdot \mathrm{s}^{-1}$. La relation d'étalonnage liant la densité de flux Ju à l'index de flux K, paramètre adimentionnel directement obtenu par le suivi des différences de température existant au niveau des 2 sondes du capteur, a été déterminée au laboratoire (Do, 1986). La surface S est déduite de relations statistiques, établies lors de l'étude in situ des arbres et de leurs systèmes racinaires. Pour chaque organe considéré (racine, tronc), elles lient cette surface au diamètre total de l'organe. Dans le cas étudié, toute la section du bois ayant été trouvée conductrice lors d'expériences de circulation sous pression d'un liquide coloré, l'expression du flux est :

$$
U=\left[\frac{K}{\alpha}\right]^{1 / \beta} \pi R^{2}
$$

où $R$ est le rayon du bois conducteur en $m, \alpha$ et $\beta$ les coefficients d'ajustement non linéaire de la relation $K$ (Ju).

\section{Dispositif de mesure}

Un capteur est placé dans chacune des 14 racines primaires de l'arbre, après creusement sous celui-ci 
d'une excavation de $60 \mathrm{~cm}$ de rayon et de $60 \mathrm{~cm}$ de profondeur, excavation dont l'axe central est celui du tronc de l'arbre. Trois fluxmètres sont placés dans la même section du tronc. Leur insertion, sur des génératrices dont la distance angulaire est de $120^{\circ}$, a conduit à affecter un tiers de la surface conductrice totale du tronc à chaque fluxmètre. Le positionnement de ces 3 génératrices est tel qu'à chaque secteur du tronc ainsi défini on peut relier, en fonction de l'insertion des différentes racines sur le tronc, un faisceau racinaire bien individualisé. Au fluxmètre T1 correspondent les racines $R 1, R 2, R 13$ et $R 14$, au fluxmètre $T 2$ les racines $R 3$ à $R 7$ et au fluxmètre T3 les racines $R 8$ à $\mathrm{R} 12$ (fig 1). Les 3 faisceaux racinaires sont dénommés respectivement $\Sigma R T 1, \Sigma R T 2$ et $\Sigma R T 3$. Les mesures sont faites avec un pas de temps de $30 \mathrm{~min}$ sur des séquences de temps de plusieurs jours. La saisie des données est commandée par un logiciel implanté sur un compatible $P C$ qui pilote une acquisition de données SAM60-AOIP. Les flux horaires et journaliers sont respectivement donnés avec une incertitude relative inférieure à $15 \%$ et $10 \%$ (Cabibel et Do, 1991a).

\section{Hétérogénéité spatiale de l'extraction racinaire}

L'estimation de la variabilité de l'extraction racinaire selon les secteurs sol définis, dans un volume de sol dont la profondeur est fixée à $200 \mathrm{~cm}$, nécessite :

- la détermination de l'aire en surface du sol du volume de sol intéressé par l'extraction racinaire totale. Cette estimation est nécessaire pour comparer dans une même unité, en $\mathrm{mm}$ par jour, les différents flux mesurés ;

- la définition du secteur d'activité racinaire propre à une racine :

- la répartition de ces secteurs racinaires dans les secteurs sol de référence.

\section{Aire à la surface du sol du volume de sol exploré par les racines}

En posant que l'extraction racinaire totale est égale à la variation de stock d'eau dans le profil de sol considéré, cette aire est égale au rapport :

$$
\frac{\text { Extraction racinaire totale en } \mathrm{m}^{3} \cdot \mathrm{s}^{-1}}{\text { Variation de stock hydrique dans le sol en } \mathrm{m} \cdot \mathrm{s}^{-1}}
$$

Ce calcul conduit à l'estimation d'un volume cylindrique de sol, centré sur l'arbre, qui implique une extension radiale uniforme du système racinaire. Le seul facteur explicatif testé au travers de l'hétérogénéité de l'extraction racinaire devient alors la densité racinaire.

Pour une extraction racinaire journalière moyenne trouvée de $31,1 \pm 0,9$ I par jour et une variation moyenne correspondante de stock d'eau dans le sol de 2,88 mm par jour, la surface calculée est égale à $10,80 \mathrm{~m}^{2}$. Cette valeur délimite autour de l'arbre un volume cylindrique de $1,85 \mathrm{~m}$ de rayon pour $2 \mathrm{~m}$ de profondeur.

\section{Secteur d'activité racinaire élémentaire}

Le secteur de sol exploré par le chevelu d'une racine primaire dont on ne connaît que la direction initiale et le flux total extrait $\Phi_{i}$ peut être défini par les paramètres que sont:

- le vecteur racines $\mathrm{R}_{\mathrm{i}}$, matérialisé par la direction initiale de la racine primaire au départ du collet. Elle est estimée directement dans la cavité creusée sous l'arbre (figs 1 et 2). Son utilisation implique l'hypothèse d'une conservation de la direction initiale pour l'ensemble de la racine ;

- les bissectrices des angles entre les vecteurs racines, qui fixent les limites d'exploration des chevelus racinaires correspondants, chaque secteur d'exploration pour une racine étant alors défini par un secteur angulaire $\alpha_{i}$ (fig 2). La définition de ce secteur angulaire implique l'hypothèse d'une non interpénétration des chevelus racinaires.

Les 2 hypothèses émises reposent sur les observations générales faites lors de l'étude in situ des systèmes racinaires des arbres du verger ;

- la densité angulaire d'extraction, rapport du flux total $\Phi_{i}$ extrait par une racine à l'angle $\alpha_{i}$ de son secteur d'activité dans le sol. La définition de ce paramètre implique I'hypothèse d'un fonctionnement homogène de la racine dans le secteur défini.

On donne au tableau I les principales caractéristiques des 14 racines principales constituant le système racinaire de l'arbre test. La variabilité des flux entre racines apparaît importante. Le flux journalier moyen

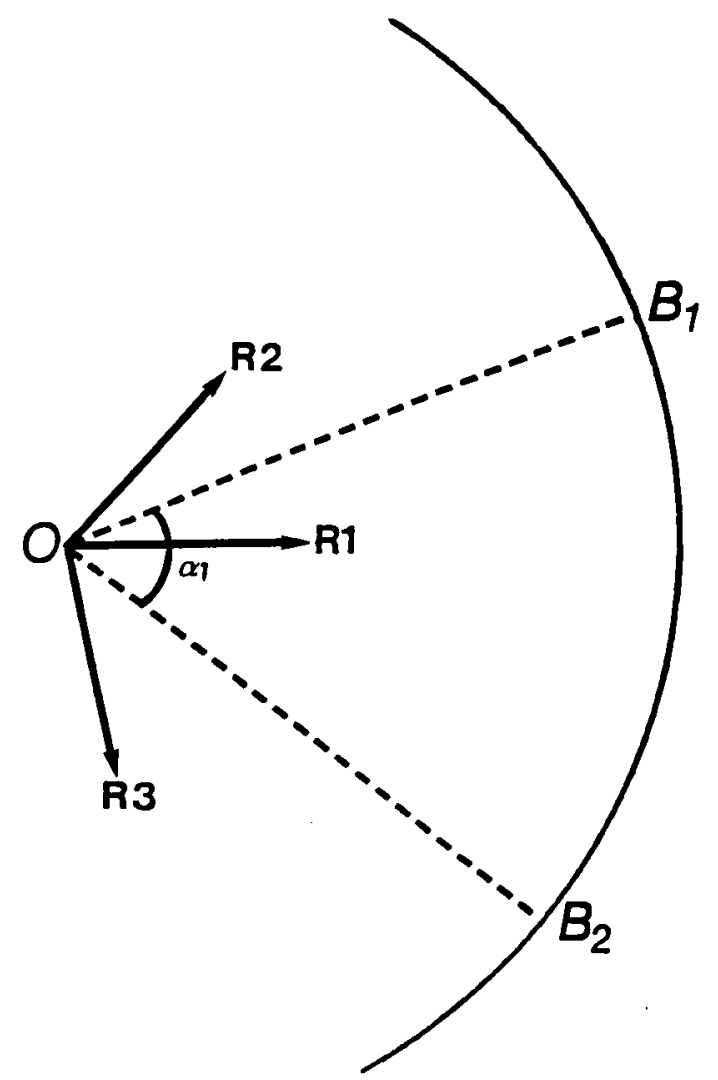

Fig 2. Secteur d'activité racinaire propre à une racine : R1, $R 2, R 3$ sont les vecteurs racinaires, $O B_{1}$ et $O B_{2}$ les limites du secteur d'activité racinaire $\alpha 1$ de R1. 
Tableau I. Caractérisation des racines primaires de l'arbre.

\begin{tabular}{|c|c|c|c|c|}
\hline $\begin{array}{l}\text { Racine } \\
N^{\circ}\end{array}$ & $\begin{array}{l}\text { Surface conductrice } \\
\qquad\left(\mathrm{cm}^{2}\right)\end{array}$ & $\begin{array}{l}\text { Flux moyen journalier } \\
\qquad\left(m^{3} \cdot 10^{-3}\right)\end{array}$ & $\begin{array}{l}\text { Extraction relative } \\
(\%)\end{array}$ & $\begin{array}{c}\text { Densité angulaire d'extraction } \\
\qquad\left(m^{3} \cdot 10^{-3} / \text { degré }\right)\end{array}$ \\
\hline 1 & 16,8 & 5,501 & 17,69 & 0,149 \\
\hline 2 & 9,5 & 3,571 & 11,48 & 0,099 \\
\hline 3 & 5,7 & 2,338 & 7,52 & 0,069 \\
\hline 4 & 3,6 & 1,167 & 3,75 & 0,058 \\
\hline 5 & 3,7 & 1,413 & 4,54 & 0,064 \\
\hline 6 & 4,2 & 1,978 & 6,36 & 0,066 \\
\hline 7 & 10,7 & 2,692 & 8,66 & 0,084 \\
\hline 8 & 7,7 & 1,845 & 5,93 & 0,092 \\
\hline 9 & 19,4 & 3,331 & 10,71 & 0,115 \\
\hline 10 & 9,2 & 2,581 & 8,31 & 0,960 \\
\hline 11 & 5,1 & 1,201 & 3,86 & 0,061 \\
\hline 12 & 5,9 & 0,968 & 3,11 & 0,081 \\
\hline 13 & 3,2 & 1,104 & 3,55 & 0,058 \\
\hline 14 & 3,2 & 1,407 & 4,52 & 0,064 \\
\hline
\end{tabular}

par racine est égal à $2,221 \pm 1,26$ litres, le coefficient de variation étant de $56,7 \%$. Parallèlement la variabilité est moindre mais reste importante au niveau de la densité angulaire d'extraction. Sa valeur moyenne est égale à $0,082 \pm 0,026 \%$ et son coefficient de variation à $31,7 \%$. Des données au tableau I on déduit, pour chacun des 8 secteurs sol de référence, une fonction puits racinaire élémentaire par sommation pondérée des flux mesurés dans chacune des racines présentes dans le secteur. Inversement, pour un secteur de sol correspondant à un faisceau racinaire donné, on calcule la variation de stock d'eau par sommation pondérée des variations de stocks mesurés dans les $8 \mathrm{sec}-$ teurs élémentaires.

\section{RÉSULTATS}

Les résultats analysés sont acquis entre le 1 et le 20 juillet 1987. L'hypothèse de continuité des transferts dans le système sol-plante repose sur l'analyse de résultats obtenus entre le 2 et le 5 juillet, période présentant des conditions optimales dans la poursuite de l'objectif visé. On dispose simultanément des mesures de flux dans l'arbre, d'ETP journalière élevées et comparables qui assurent un niveau élevé de ces flux et une stabilité inter-journalière de ceux-ci, des mesures des teneurs en eau et des potentiels de l'eau dans le sol qui permettent l'établissement de bilans hydriques précis dans le sol. De plus la période d'acquisition des données, qui est une phase continue de dessèchement du sol, permet une simplification dans l'écriture du bilan hydrique (absence de pluies et drainage) et l'utilisation de la relation $h(w)$ dans les conditions de son obtention in situ. Les transferts de sève brute dans l'arbre sont analysés durant la période du 10 au 19 juillet. Cette période correspond d'une part à une phase de dessèchement du sol (10-14 juillet) faisant suite à une réhumectation du sol sous une précipitation unique de $35 \mathrm{~mm}$ le 7 juillet d'autre part à une nouvelle phase de réhumectation du sol (15-18 juillet) due à une précipitation cumulée de $55 \mathrm{~mm}$ dont $37 \mathrm{~mm}$ le 16 juillet.

\section{Bilans hydriques dans le sol}

Les profils de variation de stock hydrique dans les 8 secteurs de mesure sont schématisés en fig 3 . Ils révèlent une forte hétérogénéité d'extraction de l'eau dans le sol, tant en ce qui concerne la quantité d'eau extraite que la localisation de cette extraction dans le profil de sol. La perte en eau est maximale $\left(4,63 \mathrm{~mm} . \mathrm{j}^{-1}\right)$ et affecte l'ensemble du profil dans le secteur nord. Elle est minimale $\left(1,99 \mathrm{~mm} \cdot \mathrm{j}^{-1}\right)$ et n'affecte que les seuls horizons $60-90 \mathrm{~cm}$ dans le secteur 5 .

\section{Hétérogénéité des flux dans l'arbre}

\section{Variabilité des flux dans le tronc}

L'analyse des cinétiques des flux traversant le tronc, estimées sur les 3 génératrices de mesure, montre qu'en période de dessèchement continu du sol (10-14 juillet) les évolutions des transferts 

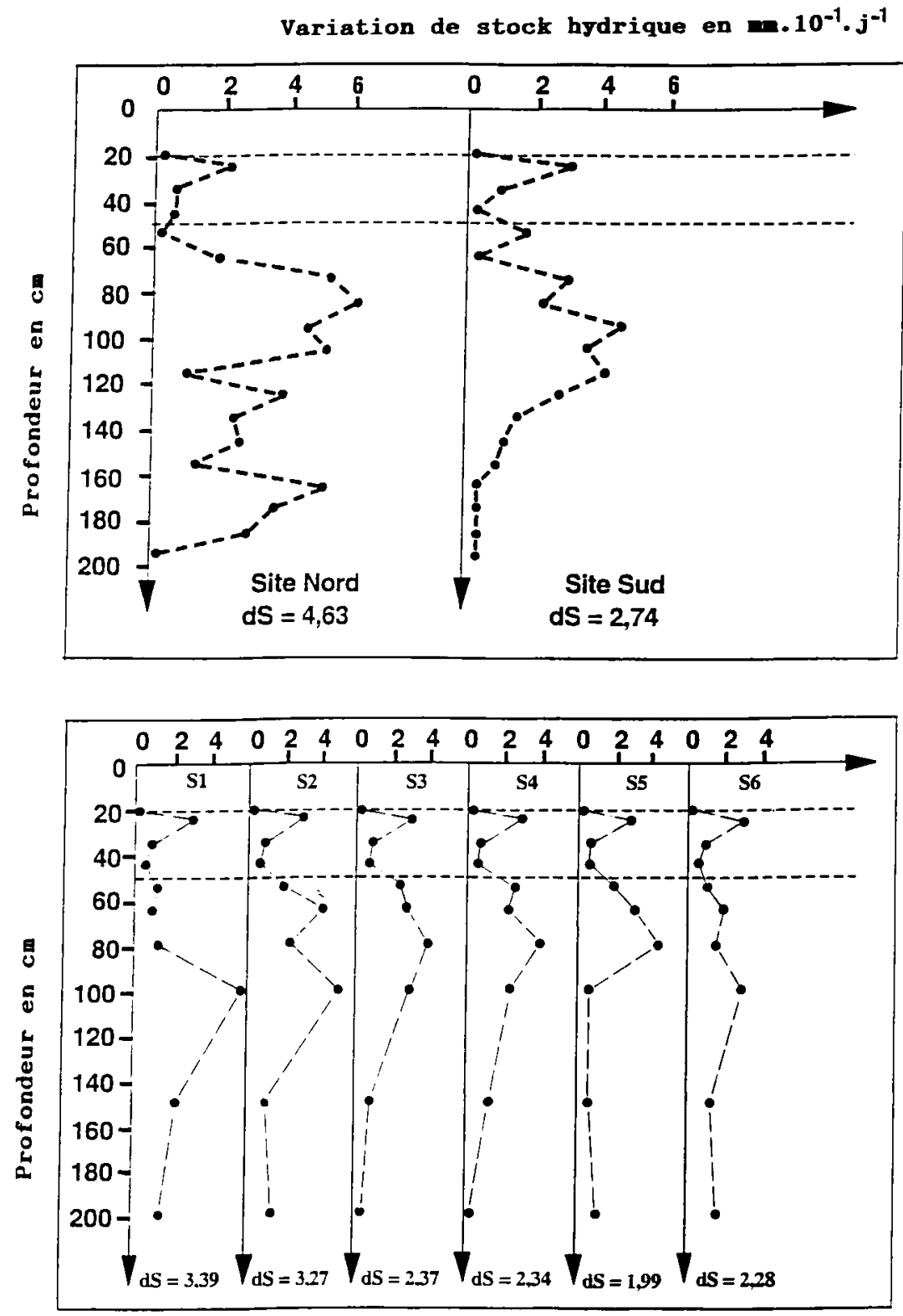

Fig 3. Variation journalière moyenne du stock hydrique dans les différents secteurs de sol. de sève brute sont généralement, au pas de temps horaire, synchrones entre elles et synchrones de l'évolution de la demande climatique s'exerçant sur le verger. Néanmoins, lors de la phase terminale d'assèchement du sol (12-14 juillet), les cinétiques de flux deviennent asynchrones des précédentes sur T2 (fig 4) et sont caractéristiques d'un état de stress hydrique. Les maxima de flux y précèdent dans le temps les maxima de demande climatique, les flux diminuant ensuite fortement alors que les ETP horaires sont encore croissantes (Cabibel et Do, 1991b).

Les flux estimés sont, quel que soit l'intervalle de temps considéré, fonction de la génératrice de mesure. On donne au tableau II, pour la période du 10 au 19 juillet, les flux journaliers et les écarts types associés, l'évolution des coefficients de variation correspondants étant donnée en figure 5A. Durant toute cette période (10-14 juillet) le sens de variation des quantités de flux est identique, les flux les plus importants apparaissant toujours au fluxmètre $\mathrm{T} 1$ et les plus faibles au fluxmètre T2. Les coefficients de variation, calculés pour les flux horaires mesurés aux heures de plus forte demande climatique (10-15 h solaires), y varient entre génératrices de $16,2 \%$ et $19,9 \%$ et, pour les flux journaliers, de $13,1 \%$ à $16,7 \%$. Il existe donc une hétérogénéité permanente des transferts hydriques dans le tronc, hétérogénéité qui affecte également les cinétiques de transfert de sève.

Le flux total est estimé, aux 2 espaces de temps considérés, par sommation des 3 quantités de flux élémentaires. Il peut apparaître cependant probable que les mesures de flux en 
Tableau II. Données climatiques ; flux mesurés sur les génératrices du tronc (T1, T2, T3 et $\bar{T})$, dans les faisceaux racinaires ( $\Sigma \mathrm{RT} 1, \Sigma \mathrm{RT} 2, \Sigma \mathrm{RT} 3$ et $\Sigma \overline{\mathrm{RT}})$ et écarts types $\sigma$.

\begin{tabular}{|c|c|c|c|c|c|c|c|c|c|c|c|c|}
\hline \multirow[t]{2}{*}{ Date } & \multirow[t]{2}{*}{$\begin{array}{c}E T P \\
\left(m m \cdot \dot{j}^{-1}\right)\end{array}$} & \multirow[t]{2}{*}{$\begin{array}{l}\text { Pluies } \\
\left(m m \cdot \dot{j}^{-1}\right)\end{array}$} & & \multicolumn{2}{|c|}{$\begin{array}{l}\text { Flux dans le tronc } \\
\qquad\left(m^{3} \cdot 10^{-3} \cdot j^{-1}\right)\end{array}$} & \multicolumn{3}{|c|}{$\sigma$} & \multicolumn{2}{|c|}{$\begin{array}{l}\text { Flux racinaire } \\
\left(m^{3} \cdot 10^{-3} \cdot j^{-1}\right)\end{array}$} & & \multirow[t]{2}{*}{$\sigma$} \\
\hline & & & $T 1$ & $T 2$ & $T 3$ & $\bar{T}$ & & $\Sigma R T 1$ & $\sum R T 2$ & $\sum R T 3$ & $\sum \overline{R T}$ & \\
\hline - & -- & & & - & - & - & - & - & - & - & - & \\
\hline $10 / 7$ & 5,6 & 0,0 & 13,76 & 10,27 & 11,56 & 11,86 & 1,77 & 12,78 & 9,13 & 11,73 & 11,22 & 1,88 \\
\hline $11 / 7$ & 5,1 & 0,0 & 13,31 & 9,65 & 11,81 & 11,59 & 1,84 & 11,95 & 8,51 & 10,74 & 10,41 & 1,74 \\
\hline $12 / 7$ & 5,5 & 0,0 & 13,64 & 9,73 & 11,82 & 11,73 & 1,96 & 12,69 & 9,03 & 11,71 & 11,14 & 1,86 \\
\hline $13 / 7$ & 5,2 & 0,0 & 13,74 & 9,87 & 11,85 & 11,82 & 1,94 & 12,64 & 9,01 & 11,56 & 11,07 & 1,08 \\
\hline $14 / 7$ & 5,1 & 0,4 & 12,23 & 9,41 & 10,69 & 10,78 & 1,41 & 10,94 & 8,78 & 9,87 & 9,86 & 0,41 \\
\hline $15 / 7$ & 2,4 & 5,4 & 8,77 & 7,81 & 7,64 & 8,07 & 0,61 & 8,16 & 7,41 & 7,51 & 7,69 & 1,29 \\
\hline $16 / 7$ & 4,4 & 36,5 & 10,46 & 9,25 & 9,01 & 9,57 & 0,78 & 11,25 & 9,05 & 8,96 & 9,76 & 1,78 \\
\hline $17 / 7$ & 4,4 & 9,3 & 11,17 & 9,32 & 8,61 & 9,71 & 1,32 & 11,77 & 9,05 & 8,41 & 9,74 & 1,62 \\
\hline $18 / 7$ & 2,4 & 4,0 & 8,47 & 6,73 & 5,67 & 6,96 & 1,41 & 8,61 & 6,26 & 5,49 & 6,79 & 2,21 \\
\hline $19 / 7$ & 4,9 & 0,0 & 12,71 & 9,92 & 8,39 & 10,34 & 2,19 & 12,48 & 9,62 & 8,13 & 10,08 & 2,19 \\
\hline
\end{tabular}

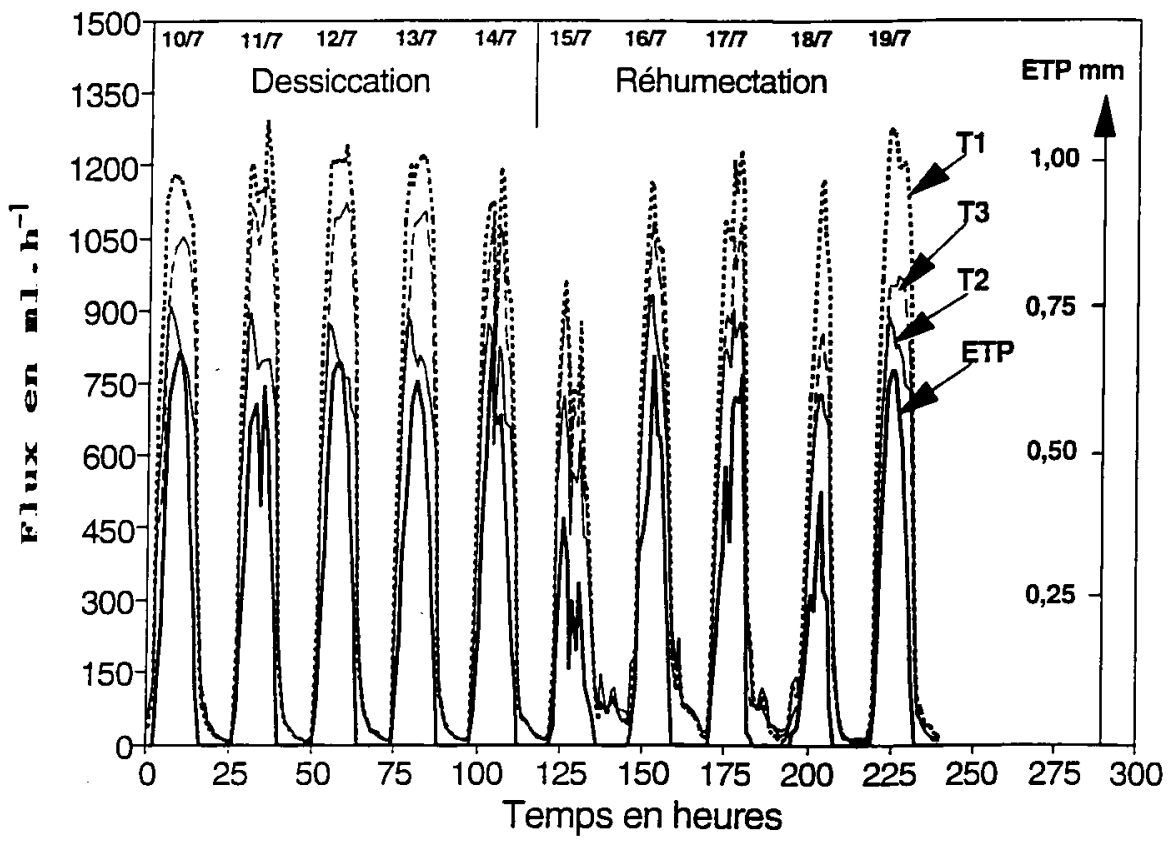

Fig 4. Cinétiques des flux horaires sur les 3 génératrices du tronc.
T1, T2 et T3 puissent être représentatives de secteurs ayant des aires conductrices variables. La solution qui aurait consisté à implanter un plus grand nombre de capteurs dans le secteur d'étude du tronc, pour mieux estimer le flux total le traversant, n'a pas été retenue. Elle n'est pas apparue forcément plus intéressante compte tenu à la fois des interférences thermiques pouvant alors se faire jour entre sondes chaudes et du peu de gain de précision pouvant être obtenu par rapport à l'intervalle de confiance estimé sur la mesure des flux (Cabibel et Do, 1991a).

Pour une génératrice de mesure donnée, les écarts de flux journaliers par rapport à la moyenne demeurent pratiquement constants. Ils sont, en moyenne, respectivement égaux à $15,4 \% \pm$ $1,2 \%,-15,3 \% \pm 2,1 \%$ et $-0,9 \% \pm 1,7 \%$ pour la période du 10 au 14 juillet en T1, T2 et T3 (fig $5 \mathrm{~B})$ et varient respectivement de 16,3 à 13,5 en $\mathrm{T} 1$, de $-17,0$ à $-12,7$ en $\mathrm{T} 2$ et de 1,9 à $-2,6$ en T3. Ils sont très supérieurs à ceux mis en évidence lors des rares études faites, sur des résineux, concernant l'hétérogénéité des transferts d'eau dans les arbres (Cohen et al, 1985 ; Granier, 1987 ; Lassoie et al, 1977). Le bois d'aubier de ce type d'arbre essentiellement composé de trachéides, éléments favorisant les transferts latéraux de sève, avait conduit certains de ces auteurs à interpréter l'hétérogénéité trouvée par une sectorisation des flux dans le tronc en conti- 

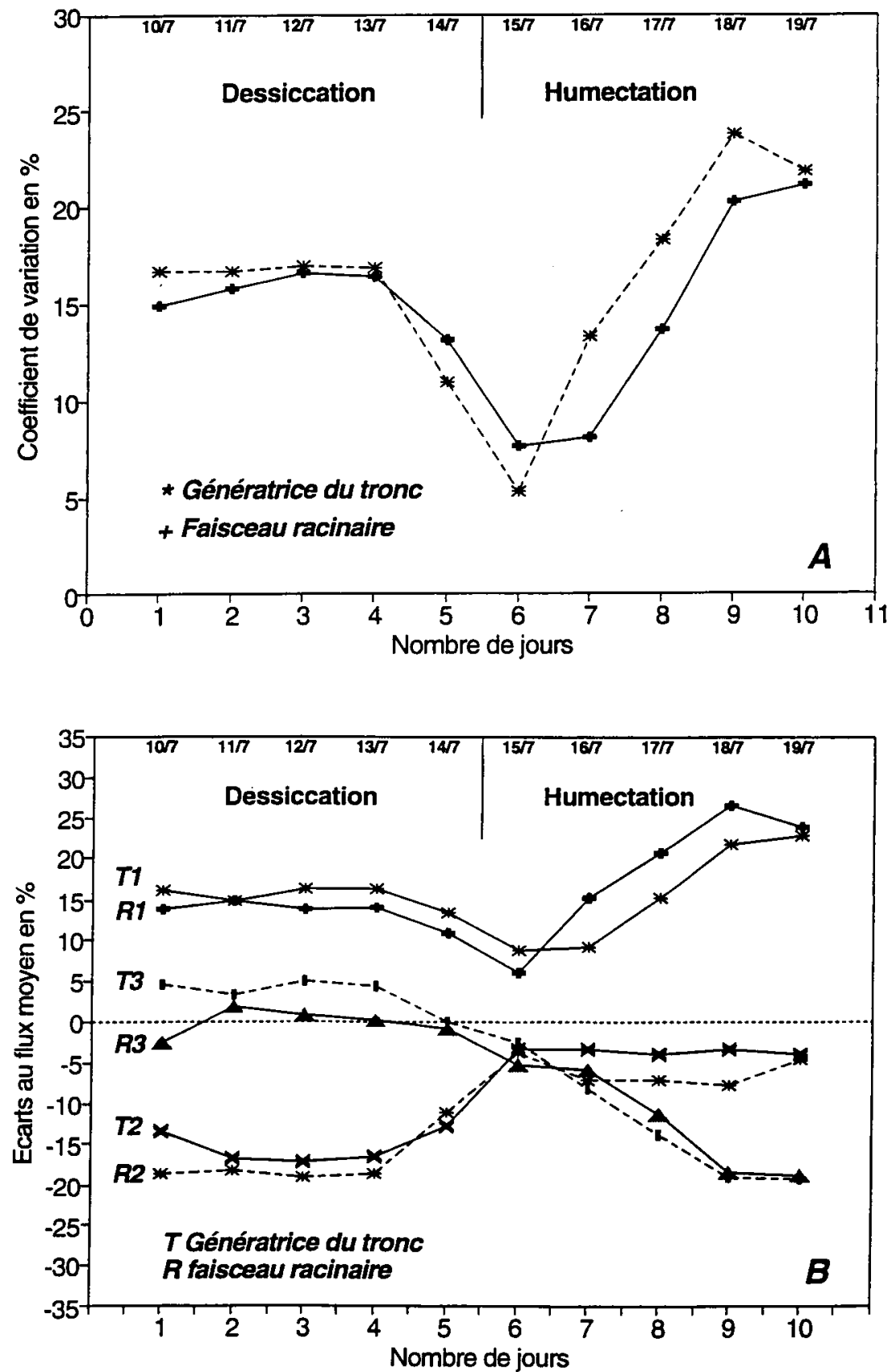

Fig 5. Coefficients de variation en $\%$ des flux journaliers de sève brute dans le tronc et les racines (A); écarts en \% au flux moyen journalier calculé dans les 2 types d'organes ligneux $(\mathrm{B})$. nuité avec une partie de la couronne foliaire (Lassoie et al, 1977). Celle-ci était supposée présenter localement des taux de transpirations variables en relation avec des taux d'insolation reçus et des activités stomatiques locales résultantes variables (Hinckley et Ritchie, 1970 ; Swanson et Lee, 1966). Nous n'avons pu retenir cette hypothèse comme origine de l'hétérogénéité des flux trouvée car :

- aucune relation n'a été mise en évidence entre l'évolution des flux horaires mesurés sur une génératrice donnée du tronc et la variation de l'incidence de l'insolation sur la fraction de la couronne foliaire lui étant apparemment associée ;
- la structure végétale plus homogène du bois d'aubier des feuillus, essentiellement composé de vaisseaux conducteurs, devrait favoriser a priori et contrairement au cas précédent une continuité des transferts de sève.

Inversement la mise en évidence d'une évolution différentielle des flux sur les 3 génératrices du tronc dès l'apparition des précipitations le 15 juillet (fig 4) nous a conduit à relier cette hétérogénéité des flux dans le tronc à l'hétérogénéité d'humectation des différents horizons du sol, donc à l'hétérogénéité de l'extraction racinaire de l'eau dans le profil de sol. En effet, après réhumectation du sol, le coefficient de variation des flux entre génératrices s'accroît comme s'accroît 
l'humectation du sol (fig 5a). Les écarts au flux moyen atteignent alors des maxima égaux à $22,9 \%,-4,0 \%$ et $-18,9 \%$ en $\mathrm{T} 1, \mathrm{~T} 2$ et $\mathrm{T} 3$, les valeurs moyennes étant respectivement de $15,6 \%$, de $-3,6 \%$ et de $-12,0 \%$ (fig $5 \mathrm{~B}$ ). Les résultats montrent que, si les flux maxima apparaissent toujours en T1, les flux minima apparaissent dès lors en T3 (tableau II) et que la participation relative des 3 flux élémentaires mesurés au flux total dans le tronc est profondément modifiée par rapport à ce qu'elle était en phase de dessiccation du sol. II apparaît donc qu'à un secteur du tronc correspondrait un faisceau racinaire et donc un secteur d'activité racinaire dans le sol, ensemble dans lequel il existerait une continuité des flux. La fonction puits moyenne dépendrait alors de l'état hydrique des divers horizons du sol explorés par les racines du faisceau considéré, cet état pouvant être modifié par la pluie de façon brutale et variable d'un secteur d'activité racinaire à l'autre.

\section{Flux traversant les racines}

Par sommation des flux élémentaires mesurés dans chacune des racines, on a estimé, aux échelles horaire et journalière, la fonction puits racinaire totale de l'arbre et celle de chacun des 3 faisceaux racinaires $\Sigma \mathrm{RT} 1, \Sigma \mathrm{RT} 2$ et $\Sigma \mathrm{RT} 3$.

\section{Flux relatifs aux faisceaux racinaires}

Pour la période du 10 au 19 juillet, on donne, au tableau II, les flux journaliers trouvés dans chaque faisceau, le flux moyen journalier correspondant et l'écart type associé. On donne en figures $5 \mathrm{~A}$ et $5 \mathrm{~B}$ l'évolution du coefficient de variation de ces flux entre secteurs racinaires et celles des écarts à la moyenne pour chacun des secteurs.

Il apparaît que, sur toute la période de dessiccation du sol et pour une demande climatique donnée, les flux racinaires journaliers estimés sont variables et fonction du faisceau considéré. Ils sont toujours plus importants pour le faisceau $\Sigma R T 1$ et les plus faibles pour $\Sigma R T 2$. Le coefficient de variation entre secteurs racinaires varie de $11,0 \%$ à $17,0 \%$ (fig $5 \mathrm{~A}$ ). Durant cette même période les écarts de flux de chacun des faisceaux racinaires au flux moyen demeurent pratiquement constants. Ils sont respectivement égaux en moyenne à $13,6 \%$ pour $\Sigma R T 1$, à $-17,1 \%$ pour $\Sigma \mathrm{RT} 2$ et à $3,5 \%$ pour $\Sigma \mathrm{RT} 3$ (fig $5 \mathrm{~B})$.

L'évolution de ces coefficients, en période de réhumectation du sol (15-18 juillet), est compa- rable à celle trouvée pour les génératrices correspondantes du tronc (figs $5 \mathrm{~A}$ et $5 \mathrm{~B}$ ). Cette concordance de comportement renforce l'hypothèse d'une continuité des flux dans le système faisceaux racinaires - génératrices du tronc.

\section{Fonction puits racinaire totale}

En phase de dessèchement du sol, elle est, à l'échelle journalière, inférieure, mais non significativement, au flux total circulant dans le tronc (fig 6A). L'écart moyen journalier étant égal à $2,42 \mathrm{I}$, l'extraction racinaire est inférieure en moyenne de $7,6 \%$ au flux mesuré dans le tronc. Cette différence peut trouver son origine dans la participation des réserves en eau de l'arbre à l'évapotranspiration (Katerji et al, 1982), notamment aux heures de plus forte demande climatique lorsque l'extraction racinaire devient limitante. Ces réserves étant situées dans les parties ligneuses de l'arbre, ce flux additionnel deviendrait essentiellement détectable au niveau du tronc. Cette hypothèse se trouve confortée par l'accroissement des écarts trouvés entre les flux horaires estimés pour les 2 types d'organes entre 12 et $17 \mathrm{~h}$ solaire, les maxima d'extraction racinaire horaire étant atteints antérieurement aux maxima de flux dans le tronc (fig 6B), les flux racinaires diminuant alors que les flux dans le tronc croissent ou demeurent élevés.

En phase de réhumectation du sol les 2 types de flux deviennent équivalents (fig 6A). Ce phénomène est le fait d'un plus grand synchronisme entre les flux diurnes racinaires et la demande climatique, tout au long de celle-ci, et de flux nocturnes racinaires importants de reconstitution des réserves de l'arbre précédemment mobilisées (fig $6 \mathrm{~B})$, l'eau ayant une plus grande disponibilité dans le sol.

Le flux hydrique qui circule du sol vers l'atmosphère au travers de l'arbre est ainsi modifié par les variations de la teneur en eau du réservoir ligneux. Dans le cas analysé, les réserves en eau de l'arbre comprises entre les sondes chaudes placées dans le tronc et celles placées dans les racines paraissent jouer un rôle important que la mesure simultanée des flux dans le tronc et les racines permet d'estimer.

\section{Continuité des flux dans le système sol-plante}

La démarche suivie a consisté à vérifier que les flux issus d'une variation de stock d'eau dans un 

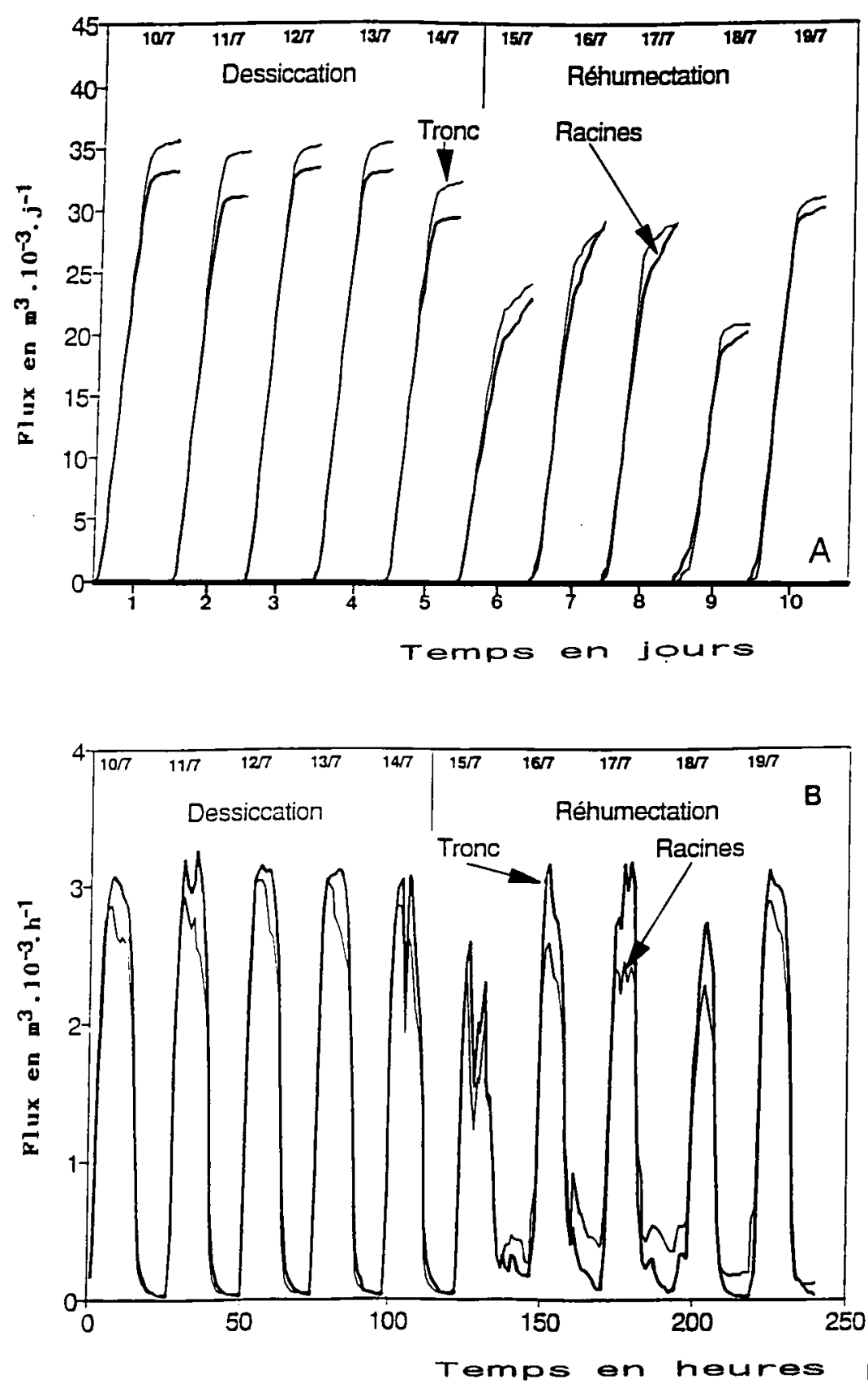

Fig 6. Flux cumulés par jour (A) et cinétiques des flux horaires $(B)$ dans le tronc et le système racinaire.

secteur donné du sol correspondaient à ceux du faisceau racinaire contenu dans ce secteur et de la génératrice du tronc en relation avec ce faisceau. Les différents flux nécessaires à la comparaison de leurs répartitions spatiales sont donnés, exprimés en mm par jour, en figures $7 \mathrm{~A}$ et 7B. II apparaît une bonne correspondance locale entre la répartition spatiale de l'extraction racinaire et la répartition spatiale de la variation de stock hydrique dans le sol. Les seuls écarts significatifs observés sont, dans les 2 cas, gommés par la fusion des secteurs juxtaposés, Ss et S2 d'une part et Sn et S5 d'autre part (fig 7A). Ces divergences peuvent être le fait d'une mau- vaise vérification de l'hypothèse de continuité directionnelle au niveau des 2 seules racines $R 2$ (secteurs Ss et S2) et R9 (secteurs Sn et S5). Lors de l'étude in situ des systèmes racinaires des arbres du verger quelques racines avaient été en effet trouvées se développer dans des secteurs angulaires de sol de $60^{\circ}$. Néanmoins ces résultats impliquent qu'il existe une continuité locale des transferts entre un secteur du sol et le faisceau racinaire qu'il contient.

La comparaison des répartitions spatiales des flux dans les 3 faisceaux de racines et les 3 génératrices correspondantes du tronc vérifie la continuité des transferts dans cet ensemble 

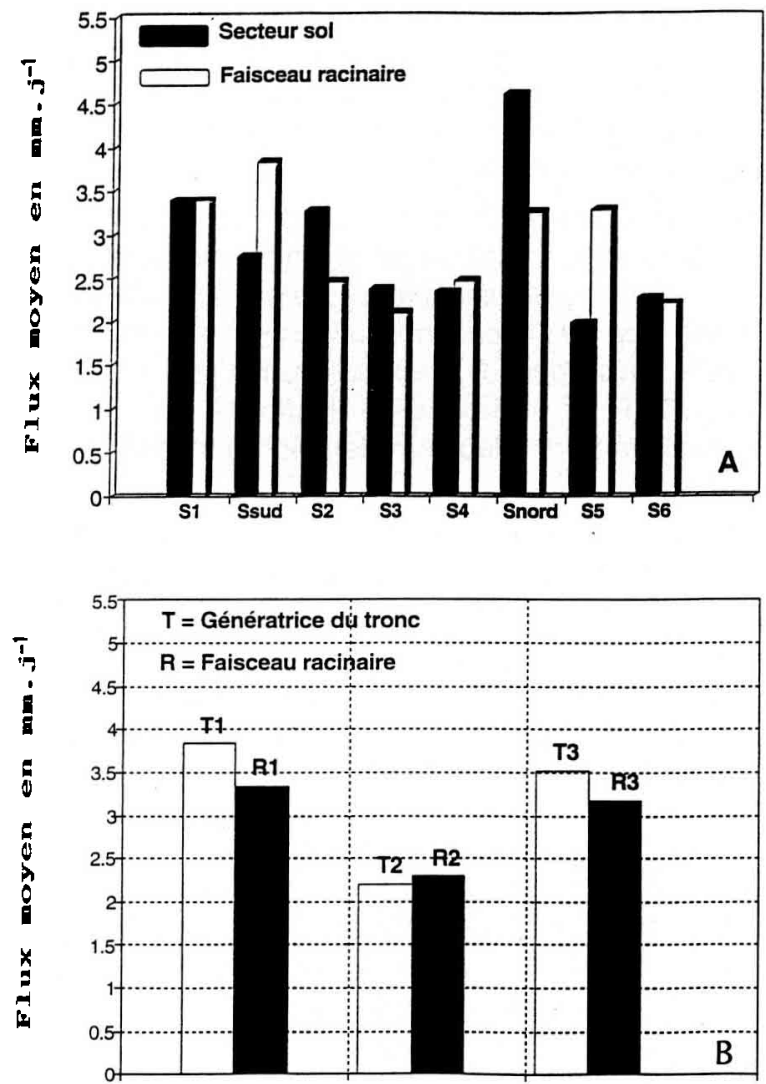

Fig 7. Flux dans les secteurs sol et les faisceaux racinaires correspondants $(A)$, dans les faisceaux racinaires et les génératrices du tronc correspondantes $(B)$.

racines-secteurs du tronc (fig 7B). La pérennité de cette relation entre les 2 structures de flux, après précipitations naturelles, conforte la réalité de cette continuité des flux dans le système sol-plante.

\section{CONCLUSIONS}

Les résultats obtenus dans l'estimation et la localisation des flux dans le système sol-arbre permettent les conclusions suivantes.

II existe, dans le tronc, une hétérogénéité des flux qui peut être importante en ce qui concerne leur amplitude et leur cinétique dans le temps et qui peut être reliée à l'hétérogénéité des flux existant dans les divers faisceaux racinaires en relation avec les secteurs du tronc.

L'amplitude de cette hétérogénéité dans le tronc est essentiellement commandée en sol hydriquement homogène par l'hétérogénéité de densité racinaire, et en sol hydriquement hétérogène par l'hétérogénéité d'humectation locale des différents horizons du sol, que celle-ci pro- vienne de dessèchements locaux importants dus à des prélèvements racinaires ou de réhumectation sous des apports d'eau.

II existe une continuité locale des flux dans le système sol-plante, les répartitions spatiales des flux mesurés dans différents secteurs du sol, les faisceaux racinaires et les génératrices du tronc leurs correspondant étant bien corrélées.

Ces conclusions conduisent à supposer que le fonctionnement racinaire et les transferts de sève brute dans les arbres fruitiers peuvent être profondément modifiés sous apports d'eau ponctuels, et pour un tel type d'irrigation, par les différentes modalités de son application à la culture. L'étude des transferts, dans les différentes parties de l'arbre, s'avère dès lors nécessaire pour parvenir à une optimisation dans l'utilisation de l'eau apportée en localisation. La fluxmétrie thermique qui permet d'appréhender simultanément et en continu l'ensemble de ces transferts constitue en ce sens un apport déterminant dans l'approche de cette optimisation.

\section{RÉFÉRENCES}

Bon M (1986) Caractérisation hydrodynamique d'un sol gonflant: Application à l'irrigation localisée. Diplôme de maîtrise d'hydrogéologie, USTL, Montpellier, France

Cabibel B (1978) Influence du mode d'irrigation sur la structure et le fonctionnement du système racinaire de jeunes pommiers. Cr Acad Agric Fr, 616-624

Cabibel B (1991) Mesures thermiques des flux de sève et comportement hydrique des arbres. III. Influence sur les flux de sève des modalités d'apport d'eau en irrigation localisée sur sol fissuré. agronomie 11 , 877-887

Cabibel B, Do F (1991a) Mesure thermique des flux de sève et comportement hydrique des arbres. I. Analyse théorique des erreurs sur la mesure des flux et validation des mesures en présence de gradients thermiques extérieurs. agronomie 11, 669-678 -

Cabibel B, Do F (1991b) Mesures thermiques des flux de sève et comportement hydrique des arbres. II. Évolution dans le temps des flux de sève et comportement hydrique des arbres en présence ou non d'une irrigation localisée. agronomie 11, 757-766

Cohen Y, Kelliher FM, Black TA (1985) Determination of sap flow in Douglas fir trees using the heat pulse technique. Can J For Res 15, 422-428

Do $F$ (1986) Estimation des flux racinaires et du comportement hydrique du pommier sous irrigation localisée en sol fissuré. Mémoire de fin d'études, ENITA, Bordeaux, France

Granier A (1985) Une nouvelle méthode pour la mesure des flux dans le tronc des arbres. Ann Sci For 42, 193-200 
Granier A (1987) Mesure du flux de sève brut dans le tronc du douglas par une nouvelle méthode thermique. Ann Sci For 44, 1-11

Habib R (1978) Absorption du 32P dans le cadre d'un essai d'irrigation localisée sur verger de pommiers. Mémoire de DEA d'agronomie, option pédologie, USTL, ENSAM, INRA, Montfavet, France

Hinckley TM, Ritchie GA (1970) Within-crown patterns of transpiration, water stress, and stomatal activity in Abies amabilis. Forest Sci 16, 490-492

Katerji N, Durand R, Hallaire M, Perrier A (1982) Modèle de transfert d'eau dans la plante et estimation des paramètres en conditions naturelles. $C R$ Acad Sci, série III, 57-62

Lassoie JP, Scott DR, Fritschen LJ (1977) Transpiration studies in Douglas fir using the heat pulse technique. For Sci 23, 377-390

Lo Cascio B (1980) Effects of 3 irrigation methods on tomato root distribution. In : Séminaires sur lirriga- tion localisée. III. Influence de l'irrigation localisée sur la morphologie et la physiologie des racines (Institut pour l'irrigation du CNR et Institut d'hydraulique agricole de l'Université de Naples, eds), Sorrento, Italie, 191-193

Ruggiero C (1980) Root distribution of tomato plants with relation to soil water regimen and to irrigation method. In : Séminaires sur lirrigation localisée. III. Influence de l'irrigation localisée sur la morphologie et la physiologie des racines (Institut pour l'irrigation du CNR et Institut d'hydraulique agricole de I'Université de Naples, eds), Sorrento, Italie, 145154

Sanders DC, Howell TA, Hile MM, Hodges L, Phene CJ (1989) Tomato root development affected by traveling trickle irrigation rate. HortScience $24,930-$ 933

Swanson RH, Lee R (1966) Measurement of water movement from and through shrubs and trees. J For 64, 187-190 